\title{
Theoretical model and characteristics of mitochondrial thermogenesis
}

\author{
Jian-Sheng Kang ${ }^{1 \bowtie}$ \\ 1 The First Affiliated Hospital of Zhengzhou University, Zhengzhou 450052, China
}

Received: 24 February 2018 / Accepted: 22 March 2018 / Published online: 19 April 2018

\begin{abstract}
Based on the first law of thermodynamics and the thermal diffusion equation, the deduced theoretical model of mitochondrial thermogenesis satisfies the Laplace equation and is a special case of the thermal diffusion equation. The model settles the long-standing question of the ability to increase cellular temperature by endogenous thermogenesis and explains the thermogenic characteristics of brown adipocytes. The model and calculations also suggest that the number of free available protons is the major limiting factor for endogenous thermogenesis and its speed.
\end{abstract}

Keywords Mitochondria, Thermogenesis, Thermal physical model, Brown adipocyte, Electrochemical potential energy

\section{INTRODUCTION}

Mitochondria are the main intracellular sites for thermogenesis, especially the mitochondria of brown adipocytes (BA), which have been targeted for therapy to reduce obesity. However, long-standing critique (Baffou et al. 2014) and debates (Baffou et al. 2015; Chrétien et al. 2018; Kiyonaka et al. 2015; Lane 2018; Suzuki et al. 2015) exist on the ability to increase cellular temperature by endogenous thermogenesis, and a good theory of intracellular thermogenesis and temperature is lacking. In this work, based on the first law of thermodynamics and the thermal diffusion equation, the thermal physical model of a mitochondrion is deduced. We found that mitochondrial thermogenesis is a special case of the thermal diffusion equation, which satisfies the Laplace equation $\left(\nabla^{2} T=0\right)$.

\section{CELL AS A THERMAL PHYSICAL SYSTEM}

The cell is a membrane-enclosed grand canonical ensemble of systems that exchanges both heat and

$\bowtie$ Correspondence: kjs@zzu.edu.cn (J.-S. Kang) particles with its surroundings. We can use the first law of thermodynamics to show that

$U=-Q+W$,

where $U$ is the cellular internal energy; $Q$ is the heat dissipated to the surroundings, and $W$ is the work added to the system. In this equation, the negative sign means that heat flows out of the cell.

For a differential change, the relation in Eq. 1 is given by the following:

$\mathrm{d} U=-\mathrm{d} Q+\mathrm{d} W$.

For thermogenesis of BA, a cell with such a small size has limited sources for energy extraction or delivery compared with the amplitudes of heat $(\mathrm{d} Q)$ and work $(\mathrm{d} W)$. Thus, it is acceptable to claim that the change in cellular internal energy $(\mathrm{d} U)$ can be neglected.

$\mathrm{d} U \approx 0$.

Therefore, the relation (Eq. 2) is reduced to the following:

$\mathrm{d} Q=\mathrm{d} W$.

We can write the cellular work $(\mathrm{d} W)$ as the sum of various forms, such as kinetic energy and potential energy: 
$\mathrm{d} W=-p \mathrm{~d} V+F \mathrm{~d} x+\sum_{i} \mu_{i} \mathrm{~d} N_{i}+\sum_{j} \varphi_{j} \mathrm{~d} q_{j}$,

where $p \mathrm{~d} V$ is the work performed by the volume change $(\mathrm{d} V)$ under pressure $(p)$, and $F \mathrm{~d} x$ is the mechanical energy used to move a distance $(\mathrm{d} x)$ under force $(F)$. In addition to kinetic energy, potential energy contains chemical potential $(\mu)$ and electric potential $(\varphi)$ in the case of changing the numbers of particles $(\mathrm{d} N)$ or charges $(\mathrm{d} q)$.

\section{THERMOGENESIS USING ELECTROCHEMICAL POTENTIAL ENERGY}

Thermogenesis in BA is executed at the mitochondrial level. A single BA contains numerous mitochondria, which show minimal volume change and almost no motility in such a crowded space (Xie et al. 2017a, b). It is acceptable to consider that both $\mathrm{d} V$ and $\mathrm{d} x$ are equal to zero such that we can ignore the changes in kinetic energy and only consider the changes in potential energy. Thus, the relation (Eq. 5) is reduced to the following:

$\mathrm{d} W=\sum_{i} \mu_{i} \mathrm{~d} N_{i}+\sum_{j} \varphi_{j} \mathrm{~d} q_{j}$.

A mitochondrion with a large negative membrane potential has the proton-motive force (pmf) for ATP synthase as well as motive forces (mf) for other particles, such as $\mathrm{Ca}^{2+}$, among others. Thus, we can write the relation (Eq. 6) as follows:

$\mathrm{d} W=p m f \cdot \mathrm{d} H^{+}+m f_{\mathrm{Ca}^{2+}} \cdot \mathrm{d} C a^{2+}+\cdots$.

With aforesaid relations (Eqs. 4 and 7), we also ignore the transient changes in mitochondria, such as $\left[\mathrm{Ca}^{2+}\right]$ (Xie et al. 2017b), for sustained thermogenesis such that the following applies:

$\mathrm{d} Q=p m f \cdot \mathrm{d} H^{+}$.

This equation matches the fact that the co-stimulation of neurotransmitters norepinephrine (NE) and ATP can effectively convert the electrochemical potential energy stored in the mitochondrial proton gradient into heat via the mitochondrial uncoupling protein-1 (UCP1) in BA (Xie et al. 2017b).

\section{TEMPERATURE GRADIENT}

According to Fourier's law, the relation between heat flux $(U$, heat per unit time per unit area, $J / s \cdot m)$ and temperature gradient $(\nabla T, \mathrm{~K} / \mathrm{m})$ is written as follows:
$J=-\kappa \nabla T$.

Fourier's law is also stated as follows:

$\mathrm{d} Q=J A \mathrm{~d} t=-\kappa A \nabla T \mathrm{~d} t$,

where $\mathrm{d} t$ is the time interval and $A$ is the area. Equations 10 and 8 together give the following:

$\nabla T=-\frac{p m f \cdot \mathrm{d} H^{+}}{\kappa A \mathrm{~d} t}$.

We can consider that a mitochondrion with a spherical shape and radius $(r)$ has an area of $A=4 \pi r^{2}$, and $\mathrm{dH}^{+} / \mathrm{d} t$ is clearly the proton current $\left(I_{\mathrm{H}^{+}}\right)$of the mitochondrion. The thermogenic proton current is directed inward and is mediated by UCP1 $\left(I_{\mathrm{UCP} 1}\right)$ after its activation. These statements mean that we can rewrite the gradient expression (Eq. 11) for BA thermogenesis as follows:

$\nabla T=-\frac{p m f}{4 \pi \kappa r^{2}} I_{\mathrm{H}^{+}}=-\frac{p m f}{4 \pi \kappa r^{2}} I_{\mathrm{UCP} 1}$.

\section{TEMPERATURE AS A FUNCTION OF TIME}

After determining the equation for the temperature gradient, we can deduce the relation between temperature and time by applying the thermal diffusion equation with a heat source (Blundell and Blundell 2010):

$\frac{\partial T}{\partial t}=D \nabla^{2} T+\frac{H}{C}$,

where $D=\kappa / C$ is the thermal diffusivity $\left(\mathrm{m}^{2} / \mathrm{s}\right) ; \kappa$ is the thermal conductivity $(\mathrm{W} / \mathrm{m} \cdot \mathrm{K}) ; C$ is the volumetric heat capacity $\left(\mathrm{J} / \mathrm{K} \cdot \mathrm{m}^{3}\right)$, and heat is generated at a rate $H$ per unit volume $\left(\mathrm{W} / \mathrm{m}^{3}, H=P / V, P\right.$ is the power, and $V$ is the volume).

In spherical polar coordinates (Blundell and Blundell 2010), we write

$\nabla^{2} T=\frac{1}{r^{2}} \frac{\partial}{\partial r}\left(r^{2} \frac{\partial T}{\partial r}\right)$

$\frac{\partial T}{\partial r}=\nabla T$

Because pmf and $I_{\mathrm{H}^{+}}$are not functions of radius $(r)$ for a single mitochondrion, Eqs. 14 and 15 together with Eq. 12 state that the thermogenesis of a mitochondrion satisfies the Laplace equation

$\nabla^{2} T=0$.

Thus, the thermal diffusion Eq. 13 for a spherical mitochondrion reduces to the following: 
$\frac{\partial T}{\partial t}=\frac{H}{C}=\frac{P}{V C}$.

Dividing both sides of Eq. 8 by a $\mathrm{d} t$ time, we write

$P=\frac{\mathrm{d} Q}{\mathrm{~d} t}=p m f \cdot \frac{\mathrm{d} H^{+}}{\mathrm{d} t}=p m f \cdot I_{\mathrm{H}^{+}}$.

Equations 17 and 18 yield the following:

$\frac{\partial T}{\partial t}=\frac{p m f \cdot I_{\mathrm{H}^{+}}}{V C}$.

\section{DISCUSSION}

\section{Steady state versus thermogenic state}

In the resting state of BA, without stimulation of sympathetic transmitters, UCP1 is inactivated by purine nucleotides. The BA or mitochondrion has a steady state described according to Eq. 19 as follows:

$I_{\mathrm{H}^{+}}=I_{\mathrm{UCP} 1}=0$,

$\frac{\partial T}{\partial t}=0$.

In the thermogenic state, it is clear that the proton current is not zero and is mediated by the activated UCP1 such that Eq. 19 states the following:

$I_{\mathrm{H}^{+}}=I_{\mathrm{UCP} 1} \neq 0$,

$\frac{\partial T}{\partial t} \neq 0$.

Using the steady state to discuss the thermogenic state leads to an $\sim 10^{-5}$ gap between Baffou's model and well-known facts (Baffou et al. 2014). In our previous paper (Xie et al. 2017a), we noted Baffou's mistakes and properly applied Eq. 17 for theoretical estimation of the maximum rate of mitochondrial temperature change. The theoretical estimation matched well with the experimental result (Xie et al. 2017a).

\section{Thermogenic rate and capacity}

After constructing the thermogenic model as a function of time (Eq. 19), we can further discuss the thermogenic characteristics of BA, such as the thermogenic capacity of the mitochondrion and the limiting factors for BA thermogenesis.

To estimate the temperature profiles of mitochondria, we must know pmf $\cdot I_{\mathrm{H}^{+}}$in Eq. 19. Mitchell's chemiosmotic theory states the following:

$p m f=\Delta \psi-\frac{2.3 R T}{F} \cdot \Delta p H$, where $\Delta \psi$ is the electrical gradient; $\Delta p H$ is the proton gradient; $R$ is the gas constant; $T$ is the temperature in Kelvin, and $F$ is the Faraday constant. The mitochondrial pmf is $\sim 200 \mathrm{mV}$. For a single mitochondrion of BA under thermogenesis, the inward thermogenic proton current is the current of the mitoplast, which is mediated by UCP1 ( $\left.I_{\mathrm{UCP} 1}\right)$. It is known that mitoplasts typically have membrane capacitances of $0.5-1.2 \mathrm{pF}$ and proton current $\left(I_{\mathrm{UCP} 1}\right)$ densities of $60-110 \mathrm{pA} / \mathrm{pF}$ (Bertholet et al. 2017).

If defining the change rate of mitochondrial temperature $\left(\frac{\partial T}{\partial t}\right)$ as a measurement of the thermogenic capacitance in BA, by taking the proton current of mitochondrion as $100 \mathrm{pA}$ and the mitochondrial volume as $1 \mu \mathrm{m}^{3}$, we obtain a theoretical rate of mitochondrial $\frac{\partial T}{\partial t}$ of $\sim 4.8 \mathrm{~K} / \mathrm{s}$ based on Eq. 19 .

The maximum experimental thermogenic capacitance of BA is comparable to $10 \mu \mathrm{mol} / \mathrm{L} \mathrm{CCCP-induced} \mathrm{ther-}$ mogenesis (Xie et al. 2017b). However, the measured maximum rate (Xie et al. 2017a) of mitochondrial $\frac{\partial T}{\partial t}$ is $\sim 0.06 \mathrm{~K} / \mathrm{s}$, which suggests that the proton current $\left(I_{\mathrm{H}^{+}}\right)$ is a limiting factor for BA thermogenesis. For the maximum transient rate of mitochondrial $\frac{\partial T}{\partial t}$, an initial transient $\left[\mathrm{Ca}^{2+}\right]$ change in mitochondria evoked by stimulation of sympathetic transmitters (Xie et al. 2017b) should be counted (Eqs. 6 and 7), which also makes a comparable contribution.

\section{Proton pool is a limiting factor}

A proton current of $100 \mathrm{pA}$ means that a single mitochondrion consumes $6.24 \times 10^{8}$ protons per second and that a single BA with $\sim 1000$ mitochondria requires $6.24 \times 10^{11}$ proton $(\sim 1 \mathrm{pmol})$ per second. Clearly, free cellular protons are the major limiting factor for thermogenesis, which was experimentally supported by the cytosol alkylation during BA thermogenesis (Xie et al. 2017b).

In Eq. 3, the change in cellular internal energy is claimed to be negligible. For verification, we calculated the numbers of free available protons, which are $\sim 6.3 \times 10^{2}$ in a mitochondrion and $\sim 10^{5}$ in a BA with a diameter of $20 \mu \mathrm{m}$ and a cytosol $\mathrm{pH}$ of 7.4. Thus, we indeed confirmed that $\mathrm{d} U$ can be neglected for sustained thermogenesis. Additionally, $\mathrm{d} U \approx 0$ suggests that the increased mitochondrial or cellular temperatures must be balanced and compensated by selected intra-mitochondrial or intracellular energy changes, such as exergonic reactions of NADH $(52.6 \mathrm{kcal} / \mathrm{mol})$ and $\mathrm{FADH}_{2}(43.4 \mathrm{kcal} / \mathrm{mol})$, which were also experimentally supported by $\mathrm{NADH}$ and $\mathrm{FADH}_{2}$ consumption during BA thermogenesis (Xie et al. 2017b). 
Consequently, the gap between the maximum experimental $\frac{\partial T}{\partial t}$ and the theoretical $\frac{\partial T}{\partial t}$ suggests that thermogenesis of BA uses less than $1 \%$ of its thermogenic capacity. In addition, as illustrated in Fig. 1, the results demonstrated that the overall averaged $\frac{\partial T}{\partial t}$ was less than $\sim 0.005 \mathrm{~K} / \mathrm{s}$. In reality, a single BA might only consume $\sim 10^{-3}-10^{-2}$ pmol proton per second for sustained thermogenesis in BA (Fig. 1). Furthermore, depolarization of the mitochondrial membrane potential and cytosol alkylation during BA thermogenesis (Xie et al. $2017 \mathrm{~b}$ ) suggest that the value of $p m f \cdot I_{\mathrm{H}^{+}}$is a factor of self-restriction for thermogenesis.

In summary, BA and its mitochondria are heat-producing micro-machines with a high efficacy limited by free proton pools. The thermogenic model (Eq. 19) and calculations suggest that BA thermogenesis relies on hydrogen and energy sources such as glucose, water, fatty acid, $\mathrm{NADH}$, and $\mathrm{FADH}_{2}$. One mol glucose and 6 mol water together can supply 24 mol protons in the tricarboxylic acid cycle. Even if glucose is supplied at a rate (Zamorano et al. 2010) of $0.18 \mathrm{pmol} / \mathrm{h} \cdot$ cell without tens or hundreds of times the glucose uptake in BA under stimulation (Orava et al. 2011; Vallerand et al. 1990 ), it is sufficient to sustain thermogenesis in BA.

\section{A generalized thermogenic model}

$\frac{\partial T}{\partial t}=\eta \frac{p m f \cdot I_{\mathrm{H}^{+}}}{V C}$.

Finally, the thermogenic model of mitochondria (Eq. 19) can be generalized as shown in Eq. 25 by multiplying the thermogenic efficiency $(\eta)$. The thermogenesis of BA under NE and ATP co-stimulation is a special case $(\eta=1)$ for Eq. 25. In general, of the potential free energy in glucose, approximately

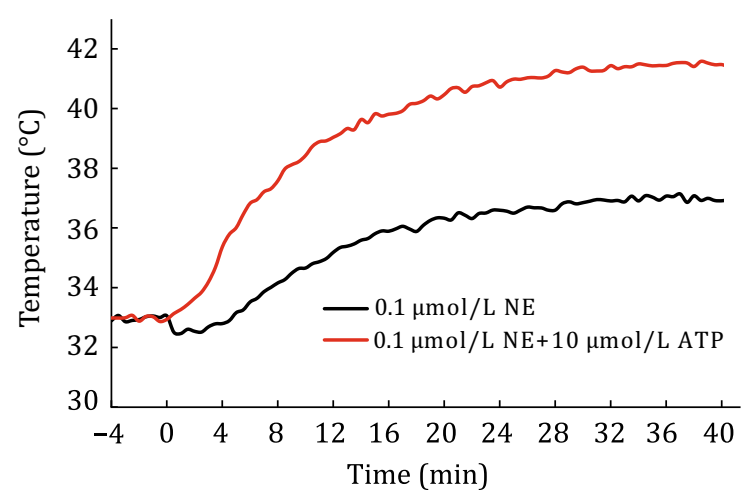

Fig. 1 Change profiles of mitochondrial temperature in BA under stimulation starting from $0 \mathrm{~min}$ of $0.1 \mu \mathrm{mol} / \mathrm{L} \mathrm{NE}$ without (black line) or with (red line) $10 \mu \mathrm{mol} / \mathrm{L}$ ATP-induced thermogenesis in BA (Xie et al. 2017b)
$40 \%$ is conserved in ATP in mitochondrial oxidative phosphorylation, and thus, the value of $\eta$ is approximately 0.6 for all other cell types except erythrocytes $(\eta=0)$, which lack mitochondria. Interestingly, NE stimulation alone activates the proton-pumping ATPase function of mitochondrial complex $\mathrm{V}$ in BA (Xie et al. $2017 \mathrm{~b}$ ), so that NE-stimulated BA show a variety of responses (heating, constant temperature or occasionally cooling) and a low efficacy of thermogenesis (Xie et al. 2017b). Consequently, the phenomena and Eq. 25 restate that the limiting factor for the capability of intracellular thermogenesis is the net proton current $\left(I_{\mathrm{H}^{+}}\right)$of proton outflow by proton pumps and proton leakage by UCP1 or other uncoupling factors.

\section{METHODS}

The thermogenic model (Eq. 19) overcomes obstacles related to the ability to increase cellular temperature by endogenous thermogenesis. Therefore, quantification is needed, which has not been performed in our previous works. Thus, the temperatures were calculated and converted from our previous data (Xie et al. 2017a, b). The calculation was based on the relation (Eq. 26) between temperature $(T)$ and the normalized intensity ratio ( $n r)$ of thermosensitive and thermoneutral mitochondrial dyes (Xie et al. 2017a).

$\frac{1}{T}-\frac{1}{T_{\text {ref }}}=-\frac{k_{\mathrm{B}}}{E_{\mathrm{a}}} \cdot \ln n r$,

where $k_{\mathrm{B}}$ is the Boltzmann constant, and $E_{\mathrm{a}}$ is the measured activation energy ( $\sim 6.55 \mathrm{kcal} / \mathrm{mol}$ ) (Xie et al. 2017a).

Acknowledgements The author thanks Dr. Xiao-Feng Liu and Dr. Tao-Rong Xie for discussions.

\section{Compliance with ethical standards}

Conflict of interest Jian-Sheng Kang declares that he has no conflict of interest.

Human and animal rights and informed consent This article does not contain any studies with human or animal subjects performed by any of the authors.

Open Access This article is distributed under the terms of the Creative Commons Attribution 4.0 International License (http:// creativecommons.org/licenses/by/4.0/), which permits unrestricted use, distribution, and reproduction in any medium, provided you give appropriate credit to the original author(s) and the source, provide a link to the Creative Commons license, and indicate if changes were made. 


\section{References}

Baffou G, Rigneault H, Marguet D, Jullien L (2014) A critique of methods for temperature imaging in single cells. Nat Methods 11:899-901

Baffou G, Rigneault H, Marguet D, Jullien L (2015) Reply to: 'Validating subcellular thermal changes revealed by fluorescent thermosensors' and 'The 105 gap issue between calculation and measurement in single-cell thermometry'. Nat Methods 12:803

Bertholet AM, Kazak L, Chouchani ET, Bogaczynska MG, Paranjpe I, Wainwright GL, Bétourné A, Kajimura S, Spiegelman BM, Kirichok Y (2017) Mitochondrial patch clamp of beige adipocytes reveals UCP1-positive and UCP1-negative cells both exhibiting Futile creatine cycling. Cell Metab 25:811-822e.4

Blundell S, Blundell KM (2010) Concepts in thermal physics. OUP, Oxford

Chrétien D, Bénit P, Ha H-H, Keipert S, El-Khoury R, Chang Y-T, Jastroch M, Jacobs HT, Rustin P, Rak M (2018) Mitochondria are physiologically maintained at close to $50{ }^{\circ} \mathrm{C}$. PLoS Biol 16:e2003992

Kiyonaka S, Sakaguchi R, Hamachi I, Morii T, Yoshizaki T, Mori Y (2015) Validating subcellular thermal changes revealed by fluorescent thermosensors. Nat Methods 12:801-802
Lane N (2018) Hot mitochondria? PLoS Biol 16:e2005113

Orava J, Nuutila P, Lidell ME, Oikonen V, Noponen T, Viljanen T, Scheinin M, Taittonen M, Niemi T, Enerbäck S, Virtanen KA (2011) Different metabolic responses of human brown adipose tissue to activation by cold and insulin. Cell Metab 14:272-279

Suzuki M, Zeeb V, Arai S, Oyama K, Ishiwata S (2015) The 105 gap issue between calculation and measurement in single-cell thermometry. Nat Methods 12:802-803

Vallerand AL, Perusse F, Bukowiecki LJ (1990) Stimulatory effects of cold exposure and cold acclimation on glucose uptake in rat peripheral tissues. Am J Physiol 259:R1043-R1049

Xie T-R, Liu C-F, Kang J-S (2017a) Dye-based mito-thermometry and its application in thermogenesis of brown adipocytes. Biophys Rep 3:85-91

Xie T-R, Liu C-F, Kang J-S (2017b) Sympathetic transmitters control thermogenic efficacy of brown adipocytes by modulating mitochondrial complex V. Signal Transduct Target Ther 2:17060. https://doi.org/10.1038/sigtrans.2017.60

Zamorano F, Wouwer AV, Bastin G (2010) A detailed metabolic flux analysis of an underdetermined network of $\mathrm{CHO}$ cells. J Biotechnol 150:497-508 